\title{
Establishment and validation of a prognostic risk classification for patients with stage T1-3NOMO esophageal squamous cell carcinoma
}

\section{Yangyu Huang}

Sun Yat-sen University Cancer Center https://orcid.org/0000-0001-5956-8803

\section{Yan Zheng}

Henan Cancer Hospital

Shen-Hua Liang

Sun Yat-sen University Cancer Center

Lei-Lei Wu

Tongji University Affiliated Shanghai Pulmonary Hospital

Xuan Liu

Sun Yat-sen University Cancer Center

Wen-Qun Xing

Henan Cancer Hospital

GuoWei Ma ( $\nabla$ magw@sysucc.org.cn )

Sun Yat-sen University Cancer Center https://orcid.org/0000-0002-5417-4941

\section{Research}

Keywords: esophageal squamous cell carcinoma, prognostic model, survival, stage T1-3NOM0, surgery

Posted Date: May 6th, 2021

DOl: https://doi.org/10.21203/rs.3.rs-414101/v1

License: (c) (i) This work is licensed under a Creative Commons Attribution 4.0 International License. Read Full License 


\title{
Establishment and validation of a prognostic risk classification for patients with stage T1-3N0M0 esophageal squamous cell carcinoma
}

\author{
Yang-Yu Huang, $\mathrm{MD}^{1 \dagger}$,Yan Zheng, $\mathrm{MD}^{2 \dagger}$,Shen-Hua Liang, $\mathrm{MD}^{1 \dagger}$,Lei-Lei $\mathrm{Wu}, \mathrm{MD}^{3}$, \\ Xuan Liu, MD ${ }^{1}$, Wen-Qun Xing, MD, PhD ${ }^{2 *}$ and Guo-Wei Ma, MD, PhD ${ }^{{ }^{*}}$
}

${ }^{1}$ Sun Yat-sen University Cancer Center, State Key Laboratory of Oncology in South China, Collaborative Innovation Center for Cancer Medicine, Sun Yat-sen University, 651 Dongfengdong Road, Guangzhou 510060, People's Republic of China

${ }^{2}$ The Affiliated Cancer Hospital of Zhengzhou University/Henan Cancer Hospital, 1 Jianshedong Road, Zhengzhou 45000, People's Republic of China

${ }^{3}$ Department of Thoracic Surgery, Shanghai Pulmonary Hospital, Tongji University School of Medicine, Shanghai, People's Republic of China

†These authors contributed equally to this work.

*These authors contributed equally to this work as senior authors.

*Corresponding author: Guo-Wei Ma, M.D.

The Department of Thoracic Surgery, Sun Yat-sen University Cancer Center, 651 Dongfengdong Road, Guangzhou 510060, People's Republic of China

Tel: +862087343317 ; Fax: +862087343392

E-mail: $\underline{\text { magw@sysucc.org.cn }}$

Co-authors' E-mail addresses:

Yang-Yu Huang: huangyy3@sysucc.org.cn; Yan Zheng: sunnyzheng1@,126.com, Shen-Hua Liang :

liangsh5@sysucc.org.cn, Lei-Lei Wu: wull79@yeah.net, Xuan Liu: liuxuan@sysucc.org.cn, WenQun Xing:1294200325@qq.com.

\section{Abstract}


Background: No existing methods can accurately predict postoperative prognosis in patients with stage T1-3N0M0 esophageal squamous cell carcinoma (ESCC). We explored the postoperative prognosis of such patients using a new prognostic risk classification system (Esorisk).

Methods: Patients with stage T1-3N0M0 ESCC from two cancer centers-Sun Yat-sen University Cancer Center (training cohort: $\mathrm{N}=819$ ) and Henan Cancer Hospital (external validation cohort: $\mathrm{N}=177$ ) —who underwent esophagectomy between 1995 and 2015 were included. We integrated significant risk factors for death events into multivariable logistic regression models and applied them to the training cohort to build Esorisk. The parsimonious aggregate Esorisk score was calculated for each patient; the training set was divided into three prognostic risk classes according to the $33 \mathrm{rd}$ and 66th Esorisk score percentiles. Esorisk's association with cancer-specific survival (CSS) was assessed using Cox proportional hazard regression analyses.

Results: The Esorisk model was: $[10+0.023 \times$ age $+0.517 \times$ drinking history $-0.012 \times$ hemoglobin $-0.042 \times$ albumin $-0.032 \times$ lymph nodes removed]. Patients were grouped into three classes - Class A (5.14-7.26, low risk), Class B (7.27-7.70, middle risk), and Class C (7.71-9.29, high risk). In the training group, the 5-year CSS decreased across categories (A: 63\%, B: 52\%, C: 30\%; log-rank $P<0.001$ ). Similar findings were observed in the validation group (A: $81 \%$, B: $69 \%$, C: $48 \%$; log-rank $P<0.001)$. Esorisk stratified the CSS in patients from two cohorts with stage pT1 $(P=0.0029)$, pT2 $(P<0.001)$, or pT3 $(P<0.001)$ disease. Cox proportional hazard regression analyses showed that the Esorisk aggregate score remained significantly associated with CSS in the training cohort $(P<0.001)$ and validation cohort $(P=0.001)$ after adjusting for $\mathrm{pT}$ stage and tumor differentiation.

Conclusion: We established and validated a novel prognostic risk classification that can predict the CSS for patients with T1-3N0M0 ESCC. This may help clinicians identify patient subgroups with poor prognosis.

Keywords: esophageal squamous cell carcinoma, prognostic model, survival, stage T1-3N0M0, surgery 


\section{Introduction}

Esophageal carcinoma (EC) is one of the most prevalent malignancies worldwide and its cause is multi-factorial. The incidence and mortality of esophageal malignancies rank ninth and sixth, respectively, in the global cancer spectrum. ${ }^{1}$ About 509,000 mortalities caused by these carcinomas were reported in 2018 alone. ${ }^{1}$ Although EC is particularly prevalent in China, Japan, South Africa, Uruguay, France, and Italy, ${ }^{2}$ more than half of the newly diagnosed EC cases occur in China, resulting in the highest mortality rate in this country.

The major histological subtypes of EC include esophageal squamous cell carcinoma (ESCC) and esophageal adenocarcinoma, with ESCC being the most common, ${ }^{3}$ accounting for more than $90 \%$ of EC cases. ${ }^{4-6}$ While research has shown that neoadjuvant therapy can improve the prognosis of patients with ESCC who underwent esophagectomy, unfortunately the postoperative prognosis for these patients remains poor. Specifically, in China, the postoperative 5-year survival rate is only $20 \%$ $40 \% .{ }^{7,8}$ In order to improve the overall survival in patients with EC, it is necessary to identify those patients with a high risk of cancer recurrence and metastasis as early as possible. An accurate staging system and individualized prognosis prediction could help clinicians to identify patients with poor $\operatorname{prognoses}^{9}$ correctly; this is essential for ensuring such patients receive a multi-disciplinary treatment regimen to improve the curative effects of treatment on EC. Existing research has identified specific factors that directly influence the occurrence and progress of EC, including the preoperative C-reactive protein/albumin ratio, ${ }^{10}$ neutrophil-to-lymphocyte ratio, ${ }^{11}$ and thoracic lymph node metastasis, ${ }^{12}$ among other factors. ${ }^{13-18}$ However, currently, there are no methods that can accurately predict postoperative prognosis in patients with stage T1-3N0M0 ESCC.

To address this issue, we developed a prognostic risk classification (referred to as Esorisk) that was based on five different parameters, including the patient's age, drinking history, number of removed lymph nodes, preoperative hemoglobin level, and preoperative level of albumin. We used patients' data from Sun Yat-sen University Cancer Center (SYSUCC, training cohort) and that from the Affiliated Cancer Hospital of Zhengzhou University/Henan Cancer Hospital (HNCH, validation cohort). Following this, we used the risk score generated by Esorisk to categorize the cohort into low- 
risk, middle-risk, and high-risk subgroups that were associated with survival outcomes. This approach provided us with a clinically applicable tool that may assist clinicians with treatment recommendations in this heterogeneous patient subgroup.

\section{Materials and Methods}

\section{Patients}

The Ethics Committee of SYSUCC approved the study's protocol (approval number: YB2016-070). The study data was recorded at the Research Data Deposit of SYSUCC for future reference, with approval number: RDDB2019000777. A total of 996 patients (819 patients from SYSUCC; 177 patients from $\mathrm{HNCH}$ ) who underwent esophagectomy at the Department of Thoracic Surgery of SYSUCC (Guangzhou, China) or HNCH (Zhengzhou, China) between April 1995 and December 2015 were enrolled retrospectively in the present study. Patients eligible for this cohort study had pathologically confirmed stage T1-3N0M0 ESCC according to the $8^{\text {th }}$ edition of the American Joint Committee on Cancer Staging Manual. The data obtained from SYSUCC were considered the training group. The data from $\mathrm{HNCH}$ were considered the external validation group. The flow chart of the study is shown in Figure 1.

\section{Surgery}

Several standard surgical approaches were used, including the Sweet (left thoracotomy and diaphragm incision), McKeown (right thoracotomy, laparotomy, and neck incision), and Ivor Lewis (laparotomy and right thoracotomy) procedures. In all patients, thoracoabdominal lymphadenectomy was performed.

\section{Follow-up}

We recommended that the patients visited the outpatient department for a follow-up examination every 3-6 months for the first 2 years, every 6 months for the next 3 years, and then every year after that. Follow-up examinations consisted of history assessment, barium esophagography, physical 
examination, chest radiography, cervical ultrasonography, abdominal ultrasonography, and neckabdomen computed tomography. If necessary, patients underwent positron emission tomographycomputed tomography, endoscopy, or both.

\section{Statistical analysis}

Statistical analysis was performed using SPSS Statistics 25.0 software (IBM SPSS, Inc., Chicago, IL, USA) and R version 3.6.2 (https://www.r-project.org/). The odds ratios with 95\% confidence intervals (95\% CIs) were calculated by multivariable logistic regression analyses. Hazard ratios (HRs) with 95\% CIs were calculated by univariable and multivariable Cox regression analyses. Multivariable analysis was performed to evaluate the effect of sex, age, number of lymph nodes removed, smoking history, drinking history, surgical approach, transthoracic laterality, pathological tumor (pT) stage, tumor grade, preoperative albumin level, and preoperative hemoglobin level on cancer-specific survival (CSS). Standard deviations were used to assess the stability of data. Variables in the univariable analysis that achieved a $P$-value of $<0.05$ were entered in the multivariable analysis. The most valuable prognostic factors were further confirmed by multivariable analysis. In addition, Kaplan-Meier (K-M) analyses and log-rank tests were used to compare survival curves between different groups. Cases were censored either at patient death or at the end of the follow-up. The selection of CSS as a primary clinical endpoint was considered the most clinically relevant.

In the present study, patient demographics and clinical characteristics were reported for the training cohort. The Esorisk scoring system for CSS was constructed using the linear predictor of the logistic model, which was derived from the training data set. The selected variables were those that achieved a $P$-value of $<0.05$ in the multivariable analysis or variables considered to be related to death events. The factors used to construct the model were not related to pathological status. The cohort was categorized into Class A (low risk), Class B (middle risk), and Class C (high risk) based on the 33rd and 66th percentiles among the risk scores in the training cohort, and a risk score cutoff was defined for classifying patients in the validation cohorts. Esorisk was applied to calculate the risk scores in the validation cohort, and patient discretization into low-risk, middle-risk, and high-risk subgroups was done using the same cutoffs defined in the training data set. A two-sided $P$-value of $<0.05$ was 
considered reflective of statistical significance.

\section{Results}

\section{Patient characteristics}

The clinical characteristics of the patients from the SYSUCC and HNCH databases are listed in Table 1. Among the 996 patients, $680(68.3 \%)$ patients were men, and $316(31.7 \%)$ were women. The patients' ages ranged from 28 to 88 years (median, 59 years). The 1-, 3-, and 4-year CSS rates were $92.0 \%, 73.0 \%$, and $49.0 \%$, respectively, and the mean time from surgery to the last censoring date was 71.42 months. During the operation, the median number of lymph nodes dissected were $18.3 \pm 13.0$ and 17.6 \pm 9.2 in the SYSUCC and $\mathrm{HNCH}$ cohorts, respectively. There was no pathological diagnosis of lymph node metastasis. None of the patients had received adjuvant or neoadjuvant cytotoxic chemotherapy, radiotherapy, or immune checkpoint inhibitors. According to the patients' records (including results of computed tomography and operation records), we accurately identified the pathological staging according to the $8^{\text {th }}$ edition of the American Joint Committee on Cancer Staging Manual. The median follow-up time was 75.0 and 57.0 months for the SYSUCC and HNCH cohorts, respectively.

\section{Construction of Esorisk}

As shown in Table 2, multivariable analyses identified the following five clinical characteristics as significant death-dependent factors in patients with ESCC: age, preoperative albumin level, drinking history, number of lymph nodes removed, and preoperative hemoglobin level. Based on the results from the training group, we constructed the Esorisk classification and tested the covariates listed in

Table 2 for their association with death events. The parsimonious Esorisk model was as follows: $10+$ $0.023 \times$ age $+0.517 \times$ drinking history $-0.012 \times$ preoperative hemoglobin level $-0.042 \times$ preoperative albumin level $-0.032 \times$ number of lymph nodes removed. We did not include factors related to the pathological status in this model. We then used the risk score generated from the above model to classify the cohort into Class A (5.14-7.26, low risk), Class B (7.27-7.70, middle risk), and Class C (7.71-9.29, high risk) depending on the 33rd (7.26) and 66th (7.70) percentiles of the Esorisk 
score. In the training group, the 5-year CSS decreased across the classes (A: $63 \%$, B: 52\%, C: $30 \%$; $\log$-rank $P<0.001$; Figure 2A). The results of the Cox regression analyses with adjustment for pT stage, tumor differentiation, and other patient-related factors revealed that the Esorisk class remained

significantly associated with CSS (Class B: HR=1.544, 95\% CI=1.129-2.111; Class C: HR=2.416, 95\% CI=1.748-3.339; $P<0.001$; Table 3).

\section{Validation of Esorisk}

In order to validate the predictive accuracy of the Esorisk classification for CSS in patients with stage T1-3N0M0 ESCC, we tested the Esorisk model independently in the validation cohort of 177 patients from $\mathrm{HNCH}$. Using the same risk score cutoff values as those identified in the training cohort allowed us to stratify the patients within the validation cohort into the low-, middle-, and high-risk subgroups. Notably, the high-risk subgroup had a significantly lower 5-year CSS than did the low-risk and middle-risk subgroups (Class A: $81 \%$, Class B: 69\%, Class C: 48\%; $P<0.001$; Figure 2B). The results of the Cox regression analyses with adjustment for $\mathrm{pT}$ stage, tumor differentiation, and other patientrelated factors revealed that the Esorisk class remained significantly associated with CSS (Class C: $\mathrm{HR}=3.113,95 \% \mathrm{CI}=1.225-7.914, P=0.019$, Table 4).

\section{Subgroup analysis}

To further explore the effects of Esorisk stratification in patients with ESCC in different T stages, we used K-M analyses to draw survival curves. As expected, the results showed that the Esorisk classification could identify the cohort with poor prognosis among patients with ESCC in every T stage (all $P<0.05$, Figure 3).

\section{Discussion}

It is well known that ESCC is associated with poor prognosis, with a 5-year overall survival rate of about $20 \%-40 \%$. The research focus is increasingly being directed at developing better treatments and improving the prognosis for patients diagnosed with ESCC. Previous studies suggested that certain factors have an effect on the prognosis of patients with ESCC; however, a systematic analysis of these 
factors as prognostic tools has not been done. ${ }^{19-24}$ In the present study, we analyzed the data of patients with ESCC from two academic institutes: SYSUCC and $\mathrm{HNCH}$. We considered the data from SYSUCC as the training group, which was analyzed by applying multivariable logistic regression and univariable and multivariable Cox regression analyses. We identified the five factors of age, preoperative albumin level, drinking history, number of lymph nodes removed, and preoperative hemoglobin level as independent factors related to death events. To construct the Esorisk model, we performed calculations using the data related to these five significant variables from patients in the training cohort (Table 2). We regarded the risk score generated by the Esorisk model as the prognostic score of patients. Our results revealed that Esorisk could stratify the patients by their prognosis, and we could identify the patient subgroup with poor prognosis after esophagectomy (Figure 2, all $P<0.001$ ). To validate Esorisk, we used the same method and cutoff values from the training cohort in the external validation cohort. As expected, the validation cohort was similarly stratified by their prognosis (Figure 2B). Together, the findings from this study support that our established and validated Esorisk model can predict the CSS for patients with stage T1-3N0M0 ESCC, which may help clinicians screen subgroups to identify patients with poor prognoses. Further exploration of the stratification power of the Esorisk classification for patients in different $\mathrm{pT}$ stages showed that Esorisk had good discriminatory power in every T stage (all $P<0.05$, Figure 3).

The selected clinical factors could be easily obtained from patients' medical records. Indeed, we were able to retrieve information on the patients' ages at the time of diagnosis from patients' medical records and obtain additional data on tumor differentiation, $\mathrm{pT}$ stage, and number of lymph nodes removed from patients' pathological reports. Preoperative levels of albumin and hemoglobin were extracted from patients' laboratory test results. The easy attainability of these five factors from patients' records was beneficial for testing our model. In addition, the areas with a high incidence of EC in China are mainly located in Henan Province, Hebei Province, the Chaoshan region of Guangdong Province, and other regions. ${ }^{25-27}$ SYSUCC is located far from $\mathrm{HNCH}$, and both cancer centers accept patients with ESCC across China. Our model was constructed using cases from SYSUCC and further validated using cases from $\mathrm{HNCH}$. We excluded patients with stage T4 ESCC and those with metastases in lymph nodes and other organs because a previous study showed that 
neoadjuvant therapy could improve the prognosis in patients who were diagnosed with metastases in lymph nodes before surgery. ${ }^{28}$

Some limitations to our study should be noted. First, the sample size of patients with ESCC was insufficient; the T stage was restricted to only $\mathrm{T} 1-3$, and the data distribution of the $\mathrm{T}$ stage was not balanced. To improve this aspect, the sample size would need to be expanded in further studies. Second, the patients' data used in this study originated from two academic institutes only, which might also affect the accuracy of our model. While the results presented herein indicated that our fivefactor prognostic model was useful for predicting the CSS for patients T1-3N0M0 ESCC postoperatively, a larger, multi-center study will be required to verify the applicability of this model. Third, this model only provides certain reference information to clinicians, not the treatment recommendations. The doctors would need to make treatment-related decisions according to the relevant guidelines and their clinical experience. Fourth, the follow-up period for the $\mathrm{HNCH}$ cohort was short. More follow-up research is needed to evaluate the prognosis for these patients. Fifth, the patients' records lacked the results of molecular diagnoses, such as information on the expression of programmed death-ligand 1, which is an effective prognostic factor that could improve prediction accuracy. ${ }^{29-31}$ Therefore, substantial research at the molecular level is needed to enhance the proposed model.

\section{Conclusions}

In conclusion, we established and validated a novel prognostic risk classification that can predict the CSS for patients with T1-3N0M0 ESCC. This valuable information may help clinicians detect subgroups of patients with poor prognoses. Moreover, we think that this model, based on patient data from two academic institutes, could easily be applied by other researchers to their own data.

\footnotetext{
Abbreviations

ESCC: esophageal squamous cell carcinoma; CSS: cancer- specific survival; EC: Esophageal carcinoma ; SYSUCC :Sun Yat-sen University Cancer Center; HNCH: Henan Cancer Hospital; HRs: Hazard ratios ; pT: pathological tumor; K-M: Kaplan-Meier; CI: confidence interval.
} 


\section{Acknowledgements}

Our sincere thanks to colleagues in the Department of Thoracic Surgery, Sun Yat-sen University

Cancer Center. Thanks to our respected pioneer, Professor Tie-Hua Rong, who oriented us to surgical oncology. Thank our patients who were willing to provide personal information for medical research; they were the best teachers for doctors.

\section{Author Contributions}

Conception and design of the work: MGW, XWQ, HYY. Provision of study materials or patients: HYY, XWQ, MGW. Acquisition of data: HYY, ZY and LX. Analysis of data: ZY, HYY and WLL. Interpretation of data: LSH, HYY. HYY and LSH drafted the manuscript; ZY, LSH and HYY substantially revised the manuscript. All authors read and approved the final manuscript.

\section{Funding}

This work was supported in part by the Guangdong Esophageal Cancer Institute Science and Technology Program [grant number M201916].

\section{Availability of data and materials}

Data from this study are available from the corresponding author upon reasonable request. The clinical data has been uploaded in the Research Data Deposit (approval number: RDDA20200001368; http://www.researchdata.org.cn/).

\section{Declarations}

\section{Ethics approval and consent to participate}

The study protocol was approved by the Ethics Committee of Sun Yat-sen University Cancer Center (Approval number: YB2016-070) and complies with the Declaration of Helsinki.

\section{Consent for publication}

Not applicable.

\section{Competing interests}

The authors disclose no conflicts of interest. 


\section{REFERENCES}

1. Bray F, Ferlay J, Soerjomataram I, Siegel RL, Torre LA, Jemal A. Global cancer statistics 2018: GLOBOCAN estimates of incidence and mortality worldwide for 36 cancers in 185 countries. CA Cancer J Clin. 2018;68:394-424. https://doi.org/10.3322/caac.21492.

2. Lin Y, Totsuka Y, He Y, et al. Epidemiology of esophageal cancer in Japan and China. $J$ Epidemiol. 2013;23:233-242. https://doi.org/10.2188/jea.je20120162.

3. Arnold M, Soerjomataram I, Ferlay J, Forman D. Global incidence of oesophageal cancer by histological subtype in 2012. Gut. 2015;64:381-387. https://doi.org/10.1136/gutjnl-2014308124.

4. Abnet CC, Arnold M, Wei WQ. Epidemiology of esophageal squamous cell carcinoma. Gastroenterology. 2018;154:360-373. https://doi.org/10.1053/j.gastro.2017.08.023.

5. Liang H, Fan JH, Qiao YL. Epidemiology, etiology, and prevention of esophageal squamous cell carcinoma in China. Cancer Biol Med. 2017;14:33-41. https://doi.org/10.20892/j.issn.2095-3941.2016.0093.

6. Chen W, Zheng R, Baade PD, et al. Cancer statistics in China, 2015. CA Cancer J Clin. 2016;66:115-132. https://doi.org/10.3322/caac.21338.

7. Feng Y, Wu N, Yan S, Wang X, Yang Y. Comparison of Ivor Lewis esophagectomy and Sweet esophagectomy for the treatment of middle-lower esophageal squamous cell carcinoma. J Thorac Dis. 2019:11:3584-3592. https://doi.org/10.21037/jtd.2019.07.68.

8. $\quad \mathrm{Yu} \mathrm{S}, \mathrm{Zhang} \mathrm{W}, \mathrm{Ni} \mathrm{W}$, et al. A propensity-score matching analysis comparing long-term survival of surgery alone and postoperative treatment for patients in node positive or stage III esophageal squamous cell carcinoma after R0 esophagectomy. Radiother Oncol. 2019;140:159-166. https://doi.org/10.1016/j.radonc.2019.06.020.

9. Donohoe CL, Phillips AW. Cancer of the esophagus and esophagogastric junction: an 8(th) edition staging primer. J Thorac Dis. 2017;9:E282-E284. https://doi.org/10.21037/jtd.2017.03.39.

10. Liu Z, Shi H, Chen L. Prognostic role of pre-treatment C-reactive protein/albumin ratio in esophageal cancer: a meta-analysis. BMC Cancer. 2019;19:1161. 
https://doi.org/10.1186/s12885-019-6373-y.

11. Pirozzolo G, Gisbertz SS, Castoro C, van Berge Henegouwen MI, Scarpa M. Neutrophil-tolymphocyte ratio as prognostic marker in esophageal cancer: a systematic review and metaanalysis. J Thorac Dis. 2019;11:3136-3145. https://doi.org/10.21037/jtd.2019.07.30.

12. Shang QX, Yang YS, Hu WP, Yuan Y, Ji AF, Chen LQ. Prognostic significance and role of thoracic lymph node metastasis based on Chinese expert consensus in esophageal cancer. Ann Transl Med. 2019;7:381. https://doi.org/10.21037/atm.2019.07.63.

13. Zheng Y, Fu S, He T, Yan Q, Di W, Wang J. Predicting prognosis in resected esophageal squamous cell carcinoma using a clinical nomogram and recursive partitioning analysis. Eur $J$ Surg Oncol. 2018;44:1199-1204. https://doi.org/10.1016/j.ejso.2018.04.011.

14. Zhang J, Luo A, Huang F, Gong T, Liu Z. SERPINE2 promotes esophageal squamous cell carcinoma metastasis by activating BMP4. Cancer Lett. 2020;469:390-398. https://doi.org/10.1016/j.canlet.2019.11.011.

15. Du X, Xu Q, Pan D, et al. HIC-5 in cancer-associated fibroblasts contributes to esophageal squamous cell carcinoma progression. Cell Death Dis. 2019;10:873. https://doi.org/10.1038/s41419-019-2114-z.

16. Gruber ES, Oberhuber G, Birner P, et al. The oncogene AF1Q is associated with WNT and STAT signaling and offers a novel independent prognostic marker in patients with resectable esophageal cancer. Cells. 2019;8(11):1357. https://doi.org/10.3390/cells8111357.

17. Wu LL, Liu X, Huang W, et al. Preoperative squamous cell carcinoma antigen and albumin serum levels predict the survival of patients with stage T1-3N0M0 esophageal squamous cell carcinoma: a retrospective observational study. J Cardiothorac Surg. 2020;15:115. https://doi.org/10.1186/s13019-020-01163-6.

18. Zhou H, Dong J, Guo L, et al. The prognostic value of B7-H6 in esophageal squamous cell carcinoma. Sci Rep. 2019;9:18122. https://doi.org/10.1038/s41598-019-54731-9.

19. Mariette C, Gronnier C, Duhamel A, et al. Self-expanding covered metallic stent as a bridge to surgery in esophageal cancer: impact on oncologic outcomes. J Am Coll Surg.

2015;220:287-296. https://doi.org/10.1016/j.jamcollsurg.2014.11.028. 
20. O'Grady G, Hameed AM, Pang TC, et al. Patient selection for oesophagectomy: impact of age and comorbidities on outcome. World J Surg. 2015;39:1994-1999. https://doi.org/10.1007/s00268-015-3072-y.

21. Xie SH, Lagergren J. Social group disparities in the incidence and prognosis of oesophageal cancer. United European Gastroenterol J. 2018;6:343-348. https://doi.org/10.1177/2050640617751254.

22. Shen $\mathrm{C}$, Liu Z, Wang Z, et al. Building CT radiomics based nomogram for preoperative esophageal cancer patients lymph node metastasis prediction. Transl Oncol. 2018;11:815824. https://doi.org/10.1016/j.tranon.2018.04.005.

23. Vendrely V, Launay V, Najah H, Smith D, Collet D, Gronnier C. Prognostic factors in esophageal cancer treated with curative intent. Dig Liver Dis. 2018;50:991-996. https://doi.org/10.1016/j.dld.2018.08.002.

24. Huang W, Wu L, Liu X, Long H, Rong T, Ma G. Preoperative serum C-reactive protein levels and postoperative survival in patients with esophageal squamous cell carcinoma: a propensity score matching analysis. J Cardiothorac Surg. 2019;14:167. https://doi.org/10.1186/s13019-019-0981-0.

25. Lin Y, Totsuka Y, Shan B, et al. Esophageal cancer in high-risk areas of China: research progress and challenges. Ann Epidemiol. 2017;27:215-221. https://doi.org/10.1016/j.annepidem.2016.11.004.

26. Tang WR, Chen ZJ, Lin K, Su M, Au WW. Development of esophageal cancer in Chaoshan region, China: association with environmental, genetic and cultural factors. Int J Hyg Environ Health. 2015;218:12-18. https//doi.org/10.1016/j.ijheh.2014.10.004.

27. Liu S, Huang B, Huang H, et al. Patrilineal background of esophageal cancer and gastric cardia cancer patients in a Chaoshan high-risk area in China. PLoS One. 2013;8:e81670. https://doi.org/10.1371/journal.pone.0081670.

28. Yang H, Liu H, Chen Y, et al; AME Thoracic Surgery Collaborative Group. Neoadjuvant chemoradiotherapy followed by surgeryversus surgery alone for locally advanced squamous cell carcinoma of the esophagus (NEOCRTEC5010): a phase III multicenter, randomized, 
open-label clinical trial. J Clin Oncol. 2018;36:2796-2803.

https://doi.org/10.1200/JCO.2018.79.1483.

29. Jiang C, Zhu Y, Tang S, et al. High PD-L1 expression is associated with a favorable prognosis in patients with esophageal squamous cell carcinoma undergoing postoperative adjuvant radiotherapy. Oncol Lett. 2019;17:1626-1634. https://doi.org/10.3892/ol.2018.9747.

30. Noske A, Möbus V, Weber K, et al. Relevance of tumour-infiltrating lymphocytes, PD-1 and PD-L1 in patients with high-risk, nodal-metastasised breast cancer of the German Adjuvant Intergroup Node-positive study. Eur J Cancer. 2019;114:76-88. https://doi.org/10.1016/j.ejca.2019.04.010.

31. Wu LL, Ma QL, Huang W, et al. A prognostic model for stratification of stage IB/IIA esophageal squamous cell carcinoma: a retrospective study. BMC Gastroenterol. 2021;21:59. https://doi.org/10.1186/s12876-021-01636-5. 


\section{FIGURE LEGENDS}

Figure 1. The flow chart of this study.

Figure 2. Application of Esorisk to refine the risk assessment in patients with stage T1-3N0M0 esophageal squamous cell cancer in two cohorts. (A) The training cohort. (B) The external validation cohort.

Figure 3. Application of Esorisk to refine the risk assessment in patients with esophageal squamous cell cancer in different stages. These included stage T1N0M0 (A), stage T2N0M0 (B), and stage T3N0M0 (C). 
Figures

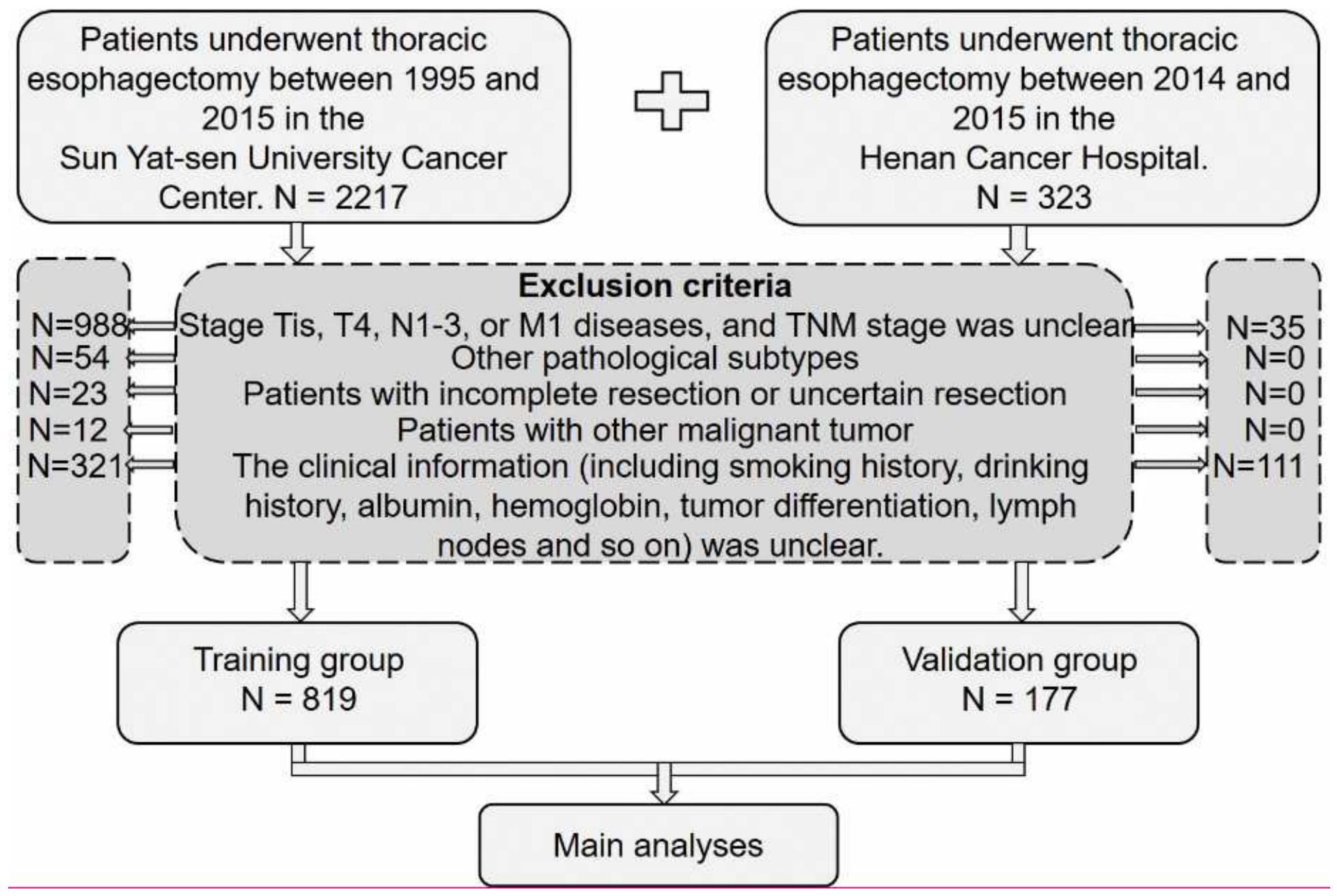

Figure 1

The flow chart of this study.

A.

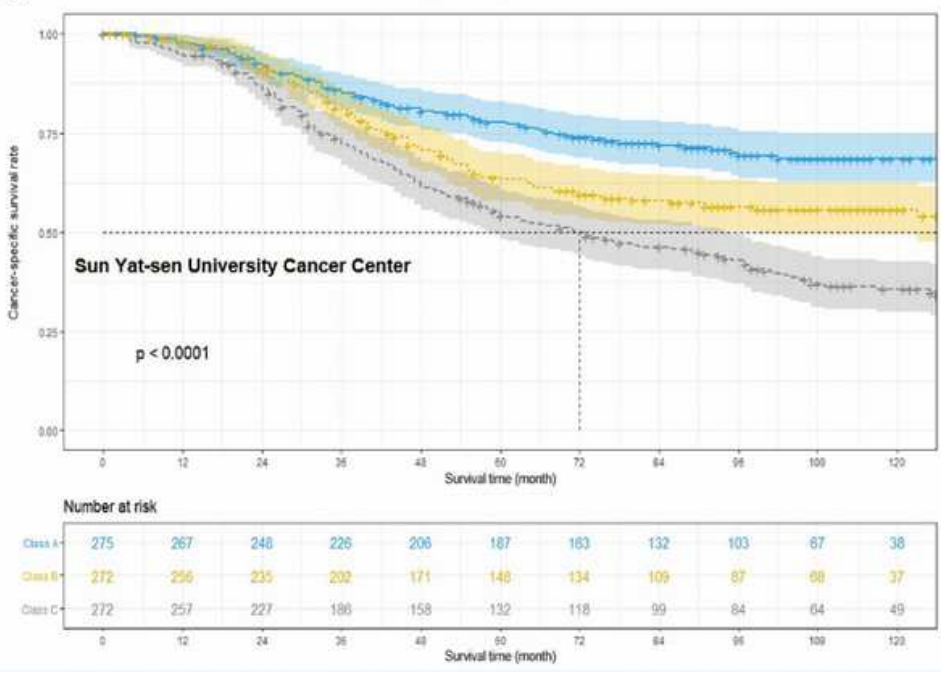

B.

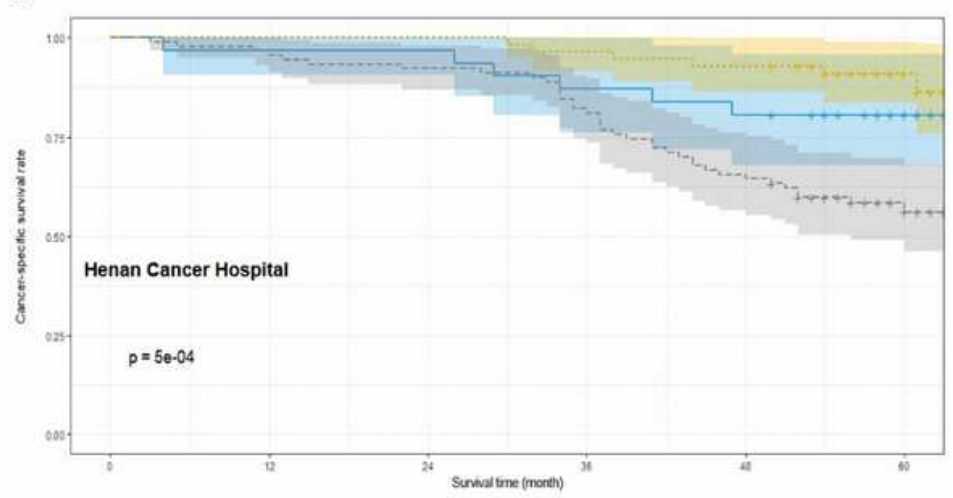

Figure 2 
Application of Esorisk to refine the risk assessment in patients with stage T1-3NOMO esophageal squamous cell cancer in two cohorts. (A) The training cohort. (B) The external validation cohort.
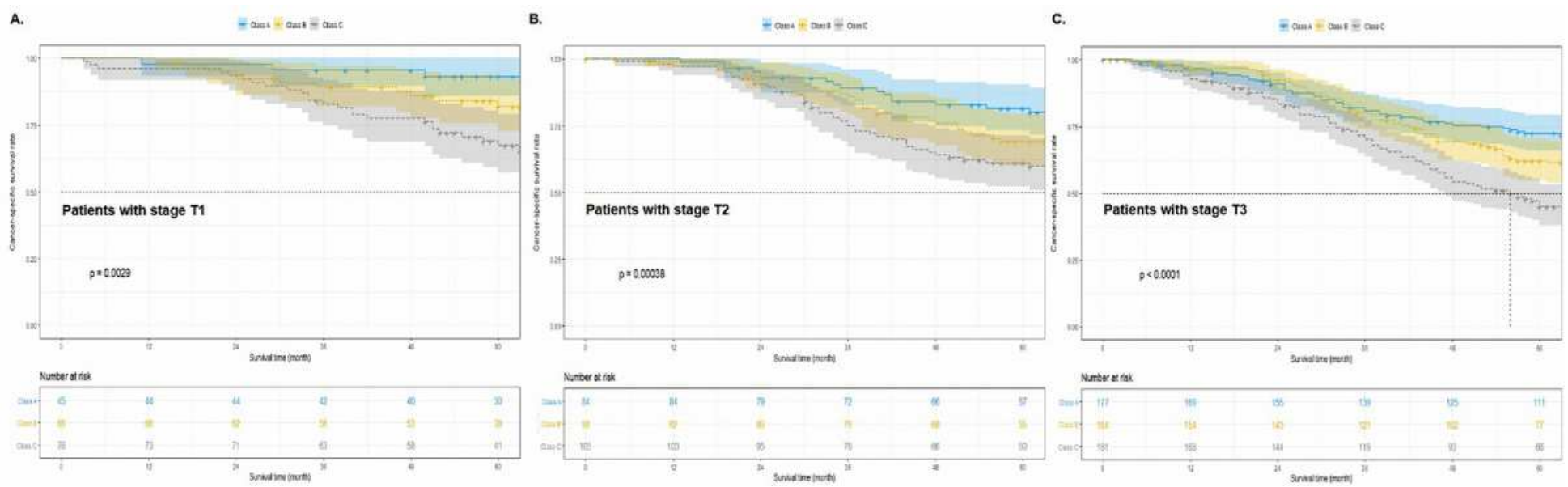

\section{Figure 3}

Application of Esorisk to refine the risk assessment in patients with esophageal squamous cell cancer in different stages. These included stage T1NOMO (A), stage T2NOMO (B), and stage T3NOMO (C). 